COLLOQUIUM MATHEMATICUM VOL. XI

\title{
ON THE EQUILIBRIUM OF SIGNED MEASURES
}

$\mathrm{BY}$

W. KLEINER (CRACOW)

\section{INTRODUCTION}

1. In 1840 Gauss [7] translated into a mathematical language the physical law which associates the equilibrium state of electric charges with sinking of the potential energy to a minimum. His arguments fell then under Weierstrass's criticism banishing them from mathematics, until a century after Gauss, Frostmann [6] recognized his great idea setting it free from time-conditioned deficiencies. Thus, modern potential theory arose to be matured in early 1940's in the works of Cartan [4, 5] and Japanese authors (Kametani and others, see [11]).

Cartan introduced the Hilbert space approach to the potential theory. Precisely, the set $\mathscr{E}$ of measures $\mu$ of finite energy (9.2) is seen to be a preHilbert space with the scalar product (9.1), energy norm $\|\mu\|$ and the corresponding strong topology. Cartan succeeded in proving that the cone $\mathscr{E}^{+} \subset \mathscr{E}$ of positive measures is complete.

2. Now the problem suggests itself to study how other norms and topologies are related to the above ones. A particularly fine result is obtained for absolute mass norm (see section 5):

$$
|\mu|(K) \leqslant c\|\mu\|
$$

for positive $\mu \subset K$ (section 4), $c$ being a positive constant depending on $K$. The square of the least possible $c$ is called capacity of $K$.

The proof is a routine one [6]. In the vaguely compact (section 6) class $\mathscr{M}_{1}=\{\mu: \mu \subset K, \mu \geqslant 0, \mu(K)=1\}$ the energy is vaguely lover semicontinuous, so $\min \|\mu\|$ is attained by an $\eta \in \mathscr{M}_{1}$, thus being positive; if we denote it by $1 / c$, then $1=|\mu|(K) \leqslant c\|\mu\|$ in $\mathscr{M}_{1}$. Now, the set of all positive measures is a cone, $i$. e. it is closed under positive linear combinations, and both the absolute mass and energy-norm are homogeneous; so, when multiplying $\mu$ by a positive constant, our last inequality is generalized to all positive measures. 
3. When we pass to signed measures, the situation changes deeply. This is most striking when we look for the literature: it simply does not exist. All we can find is the negative. The whole space $\mathscr{E}$ is not complete (section 9); the absolute mass norm is not continuous, neither in the vague nor even in the strong topology. The more, inequality (2.1) is no longer true.

Now, the problem we have set up was to give a substitute just to this inequality. It is clear that the absolute mass is then to be replaced by some other norm. But the simplest - the total mass $|\mu(K)|-$ must be refused as the resulting theorem would be useless in the most (if not exclusively) important case of vanishing total mass. Thus we had to introduce a new norm (13.3), defined for $\sigma \subset F$ ( $F$ a hipersurface) by $[\sigma]=\sup |\sigma(S)|$, where the set $S$ varies in such a manner (13.1) that if including, say, positive $\sigma$-masses lying near by the points $P, Q$ it ought to contain to some extent the negative and positive ones lying "between" $P$ and $Q$. But even with this norm, $1^{\circ}$ additional conditions should be imposed on $\sigma$ and in particular on the supporting set $F, 2^{\circ}$ a Lipschitz condition (2.1) is not available, but merely a Hölder one (Theorem VII), The set of measures allowed is no longer a cone, so the proofs become more elaborate.

The lack of interest for problems on signed measures may result not only of the unpromising look of the field, but of the probable lack of physical applications. E. g., the situation we bring forth in this paper roughly corresponds to the following. A sphere (or other regular surface) is covered with two films $S_{+}, S_{-}$, their common boundary $C$ being an insulating thread while $S_{+}$and $S_{-}$are conductors, perfectly plastic up to some definite limit. We charge $S_{ \pm}$with a charge $\pm m$ respectively and allow the electrostatic forces to shape $S_{+}$and $S_{-}$till equilibrium is established (Theorems I-IV). If we add the condition of bounded density, we see our model to belong to genuine fancy physics.

Thus we can expect no interest from physics to our work. But we hope to find some from mathematics itself - the pure and applied one. When approximating a measure $\mu$ by a sequence $\mu_{n} \rightarrow \mu$, we see the error $\delta_{n}$ $=\mu_{n}-\mu$ to be a signed measure - in most cases of mass 0 . To study the degree of convergence, or - in a later perspective - to give some numerical estimations of the accuracy obtained by the $n$-th step, some knowledge of the behaviour of signed measures seems to be indispensable. Examples of such applications will be found in the author's forthcoming papers; some of them are announced in a Conference communication (see [9])

\section{MEASURES}

4. Let $C_{0}=C_{0}(E)$ be the class of all continuous functions on a locally compact space $E$, each vanishing in some neighbourhood of infinity
(Alexandroff's point) $\infty$. By a measure $\sigma$ on $E$ we mean a functional defined for $f \in O_{0}$ and denoted by $\int f d \sigma$ (the integral of $f$ with respect to $\sigma$ ), such that $1^{\circ} \int(a f+b g) d \sigma=a \int f d \sigma+b \int g d \sigma(a, b$ arbitrary constants, $\left.f, g \in C_{0}\right)$ and $2^{\circ}$ for every compact set $K \subset E$ there is a constant $M=M(K)$ such that $\left|\int f d \sigma\right| \leqslant M \cdot \max |f|$ if $f$ vanishes outside $K$ (see [8]).

Linear combinations of measures are defined by

$$
\int f d(a \sigma+b \tau)=a \int f d \sigma+b \int f d \tau, \quad f \epsilon C_{0} .
$$

A measure $\sigma$ is positive if $\int f d \sigma \geqslant 0$ for $f \geqslant 0 ; \sigma \geqslant \tau$ if $\sigma-\tau \geqslant 0$.

The integral is extended to lower semicontinuous and then to "integrable" functions. $\varphi(x)(x \in E)$ being one of these, an equation like $d \tau=$ $=\varphi d \sigma$ means the statement on, or the definition of a measure $\tau$, namely: $\int f d \tau=\int f \varphi d \sigma\left(f \epsilon C_{0}\right)$. In particular, when $\varphi=\varphi_{A}=$ characteristic function of a Borel set $A$, the above $\tau$ is denoted by $\sigma \mid A$ and called trace of $\sigma$ on $A$. $\int f d \sigma \mid A$ is also denoted by $\int_{A} f d \sigma$. The $\sigma$-measure of $A$, or $\sigma$-mass on $A$, is defined by $\sigma(A)=\int_{A} 1 d \sigma$ - to avoid discussion: for relatively compact Borel sets $A$ only.

We write $\sigma \subset A$ if $\sigma=\sigma \mid A$; the smallest closed set with this property is called the support of $\sigma$.

5. For every $\sigma$ there exist two Borel sets $A, B$ such that $\sigma \mid A \geqslant 0$, $\sigma|B \leqslant 0, \quad \sigma=\sigma| A+\sigma \mid B$. We write $\sigma\left|A=\sigma^{+},-\sigma\right| B=\sigma^{-}$. The measure $|\sigma|=\sigma^{+}+\sigma^{-}$is called variation of $\sigma,|\sigma|(E)$ - its total variation or absolute mass, in contrast to the total mass $\sigma(E)$ (this term was used in a somewhat different meaning in the Introduction).

6. The vague topology on the linear space $M$ of measures on $E$ is defined by

$$
\sigma_{n} \rightarrow \sigma \text { means } \int f d \sigma_{n} \rightarrow \int f d \sigma \text { for all } f \in C_{0} .
$$

Every set $K(\nu)$ of measures satisfying $|\sigma| \leqslant \nu$ is vaguely compact and so is every set of bounded absolute mass.

7. If a family of functions $f(x) \epsilon C_{0}$ is given in the form $\{f(x, y)\}_{y \in E}$, then the symbol $\int f(x, y) d \sigma(x)$ is used to avoid confusion - it means $y$ to be fixed and then the integral to be taken, $f$ being now a function of $x \in \mathbb{E}$ alone. For each pair $\sigma, \tau \in M$ there exists a unique measure $\sigma \otimes \tau$ on $E \times E$ such that

(7.1) $\int f(x, y) d \sigma \otimes \tau=\int\left\{\int f(x, y) d \sigma(x)\right\} d \tau(y)=\int\left\{\int f(x, y) d \tau(y)\right\} d \sigma(x)$

for all $f(x, y) \in O_{0}(E \times E)$. This equation is then generalized to all integrable functions (Fubini's Theorem). Let us point to the equality

$$
\sigma \otimes \tau=\sigma^{+} \otimes \tau^{+}-\sigma^{+} \otimes \tau^{-}-\sigma^{-} \otimes \tau^{+}+\sigma^{-} \otimes \tau^{-}
$$


the $\sigma \otimes \tau$-integrability of $f$ means the integrability of both $f^{+}=\max \{0, f\}$ and $f^{-}=f^{+}-f$ with respect to four measures on the right side of (7.2).

\section{POTENTIAL AND ENERGY}

8. Let $E=R^{N}$ be the $N$-dimensional Cartesian linear space of points $x=\left[x_{1}, \ldots, x_{N}\right], x_{i}$ real numbers, with the usual norm $|x|=\left(x_{1}^{2}+\ldots\right.$ $\left.+x_{N}^{2}\right)^{1 / 2}$. With each $\sigma \in M$ we associate its Newton's potential

$$
U^{\sigma}(x)=\int|x-y|^{2-N} d \sigma_{y},
$$

provided it is well-defined for each $x$, the values $\pm \infty$ allowed. It is superharmonic if $\sigma \geqslant 0$. In the sequel we allow signed $\sigma$, but we limit ourselves to the case of $\sigma \subset K, K$ being a relatively compact set. Then $U^{\sigma}$ is harmonic outside the closure $K^{-}$. It is said regular at the Alexandroff's infinity $\infty$ if and only if $|x|^{N-1}\left|\operatorname{grad} U^{\sigma}(x)\right| \rightarrow 0$ as $x \rightarrow \infty[12]$; this is precisely the case if $\sigma(K)=0$. Then the limit $U^{\sigma}(\infty)=0$ is never an upper nor lower bound for $U^{\sigma}$ in any neighbourhood of infinity - thus, the maximum principle is valid even in domains containing $\infty$.

9. Let $\sigma, \tau \in M$; we put

$$
\begin{gathered}
(\sigma, \tau) \stackrel{\text { df }}{=} U^{\sigma} d \tau=\int U^{\tau} d \sigma=(\tau, \sigma), \\
\|\sigma\|^{2}=(\sigma, \sigma) .
\end{gathered}
$$

Let $\mathscr{E}$ be the set of measures $\sigma$ with both $\left\|\sigma^{+}\right\|^{2}<\infty$ and $\left\|\sigma^{-}\right\|^{2}<\infty$; then $\|\sigma\|^{2}$ is well-defined, as is $(\sigma, \tau)$ for $\sigma, \tau \in \mathscr{E}$; the second equality in (9.1) is then certainly true in virtue of Fubini's Theorem. For $\sigma$ or $\tau$ not belonging to $\mathscr{E}$ we leave off the discussion of (9.1).

If we observe that the energy $\|\sigma\|^{2}$ is strictly positive whenever $\sigma \neq \equiv([14]$, p. 6$), \mathscr{E}$ appears to be a pre-Hilbert space with the inner product (9.1), called mutual energy of $\sigma$ and $\tau$. The positive cone $\mathscr{E}^{\text {t }}$ $=\{\sigma: \sigma \epsilon \mathscr{E}, \sigma \geqslant 0\}$ is complete in the norm \|\|$[3]$. On the contrary, $\mathscr{E}$ itself is incomplete. We reproduce a simple example of Cartan. Let, in $R^{3}, \mu_{k}^{+}, \mu_{k}^{-}$be the uniform distributions of a charge 1 on the spheres $|x|=1-4^{-k}$ and $|x|=1$, respectively. Then the energy of $\mu_{k}=\mu_{t}^{+}-\mu_{k}^{-}$ is $\left(4^{k}-1\right)^{-1}$, so $\Sigma\left\|\mu_{k}\right\|$ converges, while $\sigma_{k}=\mu_{1}+\ldots+\mu_{k}$ has no limit. Indeed, let $\sigma_{k} \rightarrow \sigma$ strongly, whence vaguely ([4.], Prop. 4). Take a positive $f_{q}(x) \in C_{0}$ with $f(x)=1$ for $|x| \leqslant 1-4^{-q}, f(x)=0$ for $|x| \geqslant 1$. We have $\int f_{q} d \sigma \geqslant \int f_{q} d \sigma_{q}=q$, so condition $2^{\circ}$ of the section 4 is violated.

\section{MAJORIZED MEASURES ON REGULAR SURFAOE}

10. For the sake of simplicity we take $N=3$ from now on. The results, with obvious modifications, are valid for any $N \geqslant 3$. A few remarks coneerning this generalization are given in section 20 .
Let $F \subset R^{3}$ be a compact surface (2-dimensional triangulable manifold) of class $C^{2}$. In particular, $F$ has no double point, i. e. every $x_{0} \in F$ is contained in a set $A$, open in $R^{3}$, with $F \cap A$ homeomorph to a domain in $R^{2}$. We prepare for later use:

$1^{\circ}$ two constants $c_{1}, c_{2}>0$ such that for $x_{0} \in F$ and $r \leqslant \operatorname{diam} F$ the area of $F\left(x_{0}, r\right)=F \frown\left\{x:\left|x-x_{0}\right| \leqslant r\right\}$ satisfies the inequalities $c_{1} r^{2}$ $\leqslant\left|F\left(x_{0}, r\right)\right| \leqslant c_{2} r^{2}$

$2^{\circ}$ a $\delta_{0}>0$ such that for any $r \leqslant \delta_{0}$ and $x_{0} \in E$, the set $F\left(x_{0}, r\right)$ is a connected one. The constants in question exist because of $F$ being smooth and compact: the proof may be left to the reader.

When operating on $F$, we shall use the topology on $F$ induced from $R^{3}$ : a subset $A$ of $F$ will be said open, if it is an intersection of $F$ with an open set in $R^{3}$. Setting for any sets $A, B: A-B=\{x: x \in A, x \notin B\}$, we put $A^{\prime}$ $=F-A$ and $\partial A=A^{-} \cap\left(A^{\prime}\right)^{-}$for $A \subset F ; A^{-}$means the closure. The distance $\varrho(A, B)=\inf \{|x-y|: x \in A, y \in B\}$. We write $\varrho(x, B)$ instead of $\varrho(\{x\}, B)$.

11. Let $v \subset F^{r}$ be a positive measure $\neq 0$ with continuous potential, and

$$
K(\nu)=\{\sigma: \sigma \epsilon M,|\sigma| \leqslant \nu\}
$$

the class of measures $\sigma \subset F$ majorized by $\nu . K(v)$ is vaguely compact ([1], III $\$ 2$, Corr. 1 to Prop. 9).

LEMMA 1. Every $\sigma \epsilon K(\nu)$ has a continuous potential.

Proof. $\pm U^{\sigma}=U^{\nu \pm \sigma}-U^{\prime \prime}$; as $\nu \pm \sigma \geqslant 0, \quad U^{\sigma}$ and $-U^{\sigma}$ are lower semicontinuous (section 8).

LEMMA 2. $\|\sigma\|^{2}$ is a continuous function of $\sigma$ if considered for $\sigma \epsilon K(\nu)$.

Proof. Let $\sigma_{n} \in K(\nu), \sigma_{n} \rightarrow \sigma$; then (section 7) $\sigma_{n} \otimes \sigma_{n} \rightarrow \sigma \otimes \sigma \quad([1]$ III $\S 5$, Prop. 4). Put $f(r, \delta)$ equal to $r^{-1}$ for $r \epsilon\langle 0, \delta\rangle$, 0 for $r \epsilon\langle 2 \delta, \infty\rangle$, linear in $\langle\delta, 2 \delta\rangle$, and $g(r, \delta)=r^{-1}-f(r, \delta)$. With any $\varepsilon>0$ and suitable $\delta>0$

$$
\left|\int f(|x-y|, \delta) d \sigma_{n} \otimes \sigma_{n}\right| \leqslant \int f(|x-y|, \delta) d \nu \otimes v<\varepsilon,
$$

since $f \leqslant|x-y|^{-1}$ which is $\nu \otimes \nu$-summable and $f \rightarrow 0$ with $\delta \rightarrow 0$. The same inequality holds for $\sigma$. By section $6, \mid \int g(|x-y|, \delta) d \sigma_{n} \otimes \sigma_{n}-$ $-\int g(|x-y|, \delta) d \sigma \otimes \sigma \mid<\varepsilon$ for great $n-$ and for such an $n,\left|\left\|\sigma_{n}\right\|^{2}-\|\sigma\|^{2}\right|$ $<3 \varepsilon$.

12. $v$ may not be absolutely continuous with respect to the area on $F$, but more than mere continuity can be proved. Put for $A \subset F$

$$
\begin{gathered}
\delta A=\{x: x \in F, \varrho(x, A)<\delta\}, \\
\omega(\delta ; \nu, l)=\sup \{\nu(\delta C):|C| \leqslant l\} ;
\end{gathered}
$$


where the upper bound has to be taken for all continuous curves $C \subset F$ of finite length $|C| \leqslant l$.

LEMIMA 3. $\omega(\delta ; \nu, l) \rightarrow 0$ as $\delta \rightarrow 0$.

Proof. Assume on the contrary that $\delta_{n} \rightarrow 0$ and $\omega\left(\delta_{n} ; v, l\right)>a>0$; this implies the existence of rectifiable curves $C_{n}$ of lengths $\leqslant l$ with $\nu\left(\delta_{n} C_{n}\right)>a$. Let us represent $C_{n}$ as contractive images of a segment $Q=$ $=\langle 0, l\rangle$, i. e.

(12.3) $\quad C_{n}: \quad x=\varphi_{n}(u),\left|\varphi_{n}(u)-\varphi_{n}\left(u^{*}\right)\right| \leqslant\left|u-u^{*}\right|, \quad u, u^{*} \in Q$.

$\varphi_{n}$ are equicontinuous and $Q$ is compact, so an uniformly convergent subsequence can be drawn - we denote it again by $\varphi_{n}$, so $\varphi_{n} \rightarrow p$. By (12.3) $\varphi$ represents a rectifiable curve $C$. Its capacity $\left(^{1}\right)$ cap $C$ is known to be 0 , so to any $\varepsilon>0$ a $\delta>0$ can be given such that $\operatorname{cap}(\delta C)^{-}<\varepsilon$. Then $v\left((\delta C)^{-}\right)^{2}<\varepsilon\|v\|^{2}$. But by the uniform convergence $\delta_{n} C_{n} \subset \delta C$ for great $n$, thus $a^{2}<\nu\left(\delta_{n} C_{n}\right)^{2} \leqslant v(\delta C)^{2} \leqslant \varepsilon\|v\|^{2}, \varepsilon$ being arbitrary, which leads to a contradiction.

A more precise statement is given by Lemma 8 .

\section{THE NORM [б]}

13. Let $K$ be a disk in the $\left[u_{1}, u_{2}\right]=u$-plane: $K=\left\{u:|u| \leqslant R_{0}\right\}$, $C(K)$ the metric space of all continuous mappings $\psi$ of $K$ into $F$ with Tchebycheff's distance

$$
\left\|\psi_{1}-\psi_{2}\right\|=\max \left\{\left|\psi_{1}(u)-\psi_{2}(u)\right|: u \in K\right\}
$$
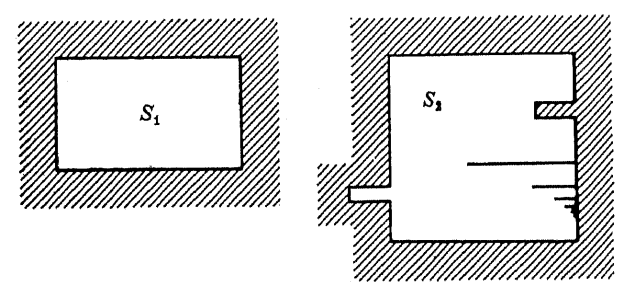

Fig. 1. Typical forms of $S \in L$.

and $\Phi_{0} \subset C(K)$ the class of all contractive one-to-one mappings of $K$ into $F$. A mapping $\psi$ is called contractive if $\left|\psi(u)-\psi\left(u^{*}\right)\right| \leqslant\left|u-u^{*}\right|$ whenever defined.

(1) The capacity of a compact set $K$ is 0 , if $\mu \subset K$ with $\|\mu\|^{2}<\infty$ implies $\mu=0$. Otherwise, $\operatorname{cap} K=\inf \left\{c: \mu(K)^{2} \leqslant c\|\mu\|^{2}\right.$ for all $\left.\mu \subset K\right\}-$ equivalently to [5], where all we need about capacity can be found.
Let $\Phi$ be the closure of $\Phi_{0}$ in $C(K)$. We call the mappings in $\Phi$ contractively nearly one-to-one. By the theorem of Ascoli ([2], X, § 4, théorème 1) $\Phi$ is a compact metric space.

It would be interesting to know whether a contractive mapping $\varphi: K \rightarrow F$, approximable in norm by one-to-one mappings, belongs necessarily to $\Phi$ or not $(\mathbf{P} 450)$.

Consider the class $L$ of images of $K$ under $\Phi$ :

With Fréchet's distance

$$
L=\{S: S=\varphi(K), \varphi \epsilon \Phi\} .
$$

$$
\varrho\left(S_{1}, S_{2}\right)=\inf \left\{\left\|\varphi_{1}-\varphi_{2}\right\|: \varphi_{i} \epsilon \Phi, \varphi_{i}(K)=S_{i}(i=1,2)\right\}
$$

$L$ becomes a compact metric space. This is easily seen when copying on $L$ the coverings of $\Phi$. Now, we assume $R_{0}$ to be such a number that $F \in L$. This is done for purely aesthetical reasons - the theory will do with any fixed $R_{0}>0$ - so we omit the existence proof.

For an $S=\varphi(K), \varphi \epsilon \Phi$, we define its contour $O S$ by

$$
C S=\varphi(\partial K) \text {. }
$$

It is a continuous rectifiable (althought not necessarily Jordan) curve of length $|C S| \leqslant l_{0}=2 \pi R_{0}$. For fixed $S$, $C S$ is not unique - it depends on $\varphi$. In what follows, we will always mean by $O S$ a concretely chosen contour.

$S$ and $C S$ are obviously closed sets.

We have $\partial S \subset C S$. Indeed, let $x_{0}=\varphi\left(u_{0}\right) \epsilon \partial S-C S$. Let $\varphi_{n} \epsilon \Phi_{0}$, $\left\|\varphi_{n}-\varphi\right\| \rightarrow 0$. Take a connected open neighbourhood $B$ of $x_{0}$ in $F$, with $B^{-} \cap C S$ void. There is an $y \in B-S$; the last set being open, it contains a closed neighbourhood of $y$. Then, for $n$ sufficiently great, $1^{\circ} \varphi_{n}(u) \neq y$ $(u \in K), 2^{0} \varphi_{n}(\partial K) \cap B^{-}$is void, $3^{\circ} x_{n}=\varphi_{n}\left(u_{0}\right) \epsilon B$. When connecting $x_{n}$ to $y$ by an are $L \subset B$, we see that $L$ has to meet $\partial \varphi_{n}(K)=\varphi_{n}(\partial K)\left(\varphi_{n}\right.$ is a topological mapping!), contrary to $2^{\circ}\left({ }^{2}\right)$.

\section{Moreover,}

(13.2) $S_{1}-S_{2} \subset \delta \partial S_{2} \subset \delta C S_{2}$ provided $S_{1}, S_{2} \in L, \varrho\left(S_{1}, S_{2}\right)<\delta$.

Indeed, $S_{1} \subset \delta S_{2}=\delta \partial S_{2} \cup S_{2} \subset\left(\delta C S_{2} \cup S_{2}\right)$ and (13.2) follows.

One could alternatively define $L$ as the class of all sets $S \subset F$ such that $\partial S$ may be covered by a curve of length $<l_{0}$. The proofs become then more elaborate, but the class $L$ is then closed under forming complements, an advantageous property bringing more symmetry in the theory (see e.g. Theorem II).

Now, we define for $\sigma \subset F$

$$
[\sigma]=\sup \{|\sigma(S)|: S \in L\} \text {. }
$$

(2) We are gratefull to Mr. J. Siciak, whose friendly criticism induced us to improve the formulation of this proof. 
This is a norm: $[a \sigma+b \tau] \leqslant|a|[\sigma]+|b|[\tau]-$ and $[\sigma]=0$ for $\sigma \equiv 0$ only. Indeed, it is sufficient to show that $[\sigma]=0$ implies $\int f d \sigma=0$ for $f$ continuous vanishing outside a small ball $B$ with centre on $F$, such functions forming an uniform base in $C_{0}(F)$ and the behaviour of $f$ outside $F$ being of no importance. Now, given an $\varepsilon>0$ cover $F \cap B$ by a finite number of disjoint small sets $S_{i} \in L\left({ }^{3}\right)$ with $M_{i}-m_{i}<\varepsilon, M_{i}$ resp. $m_{i}$ denoting the upper resp. lower bound for $f$ on $S_{i}$, and estimate

$$
\begin{aligned}
\int f d \sigma & =\sum \int f d\left(\sigma \mid S_{i}\right) \leqslant \sum\left\{M_{i} \sigma^{+}\left(S_{i}\right)-m_{i} \sigma^{-}\left(S_{i}\right)\right\} \\
& =\sum\left(M_{i}-m_{i}\right) \sigma^{+}\left(S_{i}\right)+m_{i} \sigma\left(S_{i}\right)<\varepsilon \sigma^{+}\left(F^{\prime}\right) .
\end{aligned}
$$

Letting $\varepsilon \rightarrow 0$ we obtain for all continuous functions $\int f d \sigma \leqslant 0$ and then $\int f d \sigma \geqslant 0$, since $C_{0}\left(F^{\prime}\right)$ is a linear space.

LEMMA 4. In $K(v)$, the vague convergence is uniform on $L$, i.e. if $\sigma_{n} \in K(v), \sigma_{n} \rightarrow \sigma$, then to any $\varepsilon>0$ there exists an $N$ such that $\mid \sigma_{n}(S)$ $-\sigma(S) \mid<\varepsilon(S \in L, n \geqslant N)$.

Proof. Let $\varepsilon>0$. Choose a $\delta>0$ such that $\omega\left(\delta ; \nu, l_{0}\right)<\varepsilon($ lemma 3$)$ and take a partition of unity $1 \equiv \sum_{i=1}^{r} \varphi_{i}(x)(x \in F)$, where $p_{i}$ are positive continuous and each support $F_{i}=\left\{x: p_{i}>0\right\}^{-}$is of diameter $<\delta$. Put them in such an order that $F_{1}, \ldots, F_{k-1} \subset S, F_{k}, \ldots, F_{p} \subset \delta C S, F_{p+1}, \ldots$ $F_{r} \subset S^{\prime}, S \in L$ being arbitrarily fixed. Then

$$
\begin{aligned}
& \left|\int_{S} d\left(\sigma_{n}-\sigma\right)\right| \leqslant\left|\sum_{i=1}^{k-1} \int \varphi_{i} d\left(\sigma_{n}-\sigma\right)\right|+\sum_{i=k}^{p}\left|\int \varphi_{i} d\left\{\left(\sigma_{n}-\sigma\right) \mid S\right\}\right| \\
& \leqslant \mid \text { first sum }\left|+\sum_{k}^{p} \int \varphi_{i} d 2 v<\right| \text { firstsum } \mid+2 \varepsilon
\end{aligned}
$$

since $0 \leqslant \varphi_{i}, \sum_{k}^{p} \varphi_{i} \leqslant 1$ and $v(\delta C S)<\varepsilon$. There exist an $N$ such that $\left|\int \varphi_{i} d\left(\sigma_{n}-\sigma\right)\right|<\varepsilon \mid r(n \geqslant N, i=1, \ldots, r)$, thus $\left|\int_{S} d\left(\sigma_{n}-\sigma\right)\right|<3 \varepsilon(n \geqslant N)$, q. e. d.

LEMMa 5. $[\sigma]$ is a continuous function of $\sigma$ if considered for $\sigma \epsilon K(\nu)$.

Proof. With $\sigma_{n}, \varepsilon$ and $N$ as above, ||$\left.\sigma_{n}(S)|-| \sigma(S) \|<3 \varepsilon\right\} \quad(n \geqslant N$, $S \in L)$ which implies $\left|\left[\sigma_{n}\right]-[\sigma]\right| \leqslant 3 \varepsilon$.

Remark. $[\sigma]$ is not continuous in general circumstances. Let $\sigma_{n}$ $\subset\left\{x_{n}^{+}, x_{n}^{-}\right\}, \sigma_{n}\left\{x_{n}^{ \pm}\right\}= \pm 1$, where $x_{n}^{ \pm} \in F, x_{n}^{ \pm} \rightarrow x_{0}$. Then $\sigma_{n} \rightarrow 0$, but $\left[\sigma_{n}\right]=1$

LEMrMa 6. For $\sigma \in K(v)$, the bound $[\sigma]$ is an actual maximum: $\left|\sigma\left(S_{0}\right)\right|$ $=[\sigma]$ for an $\$_{0} \in L$.

(3) Just to make obvious this possibility $F^{\prime}$ has been replaced by $F \cap B$ which can be regarded arbitrarily flat.
Proof. $L$ being compact, it is sufficient to show that for $\sigma \in K(v)$, $\sigma(S)$ is a continuous function on $L$. Indeed, given an $\varepsilon>0$ take a $\delta>0$ with $\omega\left(\delta ; v, l_{0}\right)<\frac{1}{2} \varepsilon \quad\left(l_{0}=2 \pi R_{0}\right) \quad$ (Lemma 3). Let $\varrho\left(S_{1}, S_{2}\right)<\delta$, then with $A=S_{1} \cap S_{2}$

$$
\begin{aligned}
&\left|\sigma\left(S_{1}\right)-\sigma\left(S_{2}\right)\right|=\left|\sigma\left(S_{1}-A\right)+\sigma(A)-\sigma\left(S_{2}-A\right)-\sigma(A)\right| \\
& \leqslant \nu\left(S_{1}-A\right)+\nu\left(S_{2}-A\right)<\varepsilon,
\end{aligned}
$$

since e.g. $v\left(S_{1}-A\right)=v\left(S_{1}-S_{2}\right) \leqslant v\left(\delta C S_{2}\right)<\frac{1}{2} \varepsilon$ by (13.2) and (12.2).

Lemma 7. To any $f \epsilon C^{2}(F)$, i. e. to any $f$ defined on $F$ and of class $C^{2}$ with respect to local coordinates on $F$, there exists a constant $M_{f}$ such that for every $\sigma \subset F$ we have

$$
\left|\int f d \sigma\right| \leqslant[\sigma] M_{f}
$$

In particular, for $x \notin F$ we have

$$
\left|U^{\sigma}(x)\right| \leqslant[\sigma] M(x),
$$

where $M(x)$ is a continuous function of $x \in R^{3}-F$.

Remark. We do not suppose $\sigma \in K(v)$.

Proof. Take a finite triangulation of $F$, correcting it if necessary so as to obtain $C^{2}$-sides and positive angles. Break each triangle in 3 quadrilaterals and cut them up again and again, but look after the regularity mentioned above. Thus a partition of $F$ into disjoint semi-closed quadrilaterals $F_{i}$ can be obtained with the following properties:

$$
1^{0} F=\bigcup_{i=1}^{N} F_{i}(N<\infty)
$$

$2^{\circ} F_{i}$ is a rectifiable Jordan curve, divided by four distinguished points (vertices) in four $C^{2}$-arcs (sides); if any two sides meet at a vertex, they form there an angle always $\geqslant a>0, a$ depending on the partition but not on $i$,

$3^{0} \bar{F}_{i} \frown \bar{F}_{k}$ is either void, or a common vertex, or a whole common side of $F_{i}, F_{k}(i \neq k)$,

$4^{\circ}$ any two tangent planes to $F_{i}$ form an angle $\leqslant \pi / 4$ and, moreover, $\operatorname{diam} F_{i} \leqslant \cos \pi / 4$

By projection on the tangent plane we can show that on each $F_{i}$ regular contractive curvilinear coordinates $[u, v] \epsilon Q$ can be given - precisely, $F_{i}$ can be represented by

$$
F_{i}: x=\varphi^{i}(u, v)([u, v] \epsilon Q=\langle 0,1\rangle \times\langle 0,1\rangle) \quad(i=1, \ldots, N),
$$

where $\varphi^{i}$ is defined and $\epsilon C^{2}$ in some square $Q^{*}=\langle-r, 1+r\rangle \times\langle-r$, $1+r\rangle(r>0)$ and maps it into $F$ in a contractive one-to-one way i. e. $\left|\varphi^{i}(u, v)-\varphi^{i}\left(u^{*}, v^{*}\right)\right| \leqslant\left|[u, v]-\left[u^{*}, v^{*}\right]\right|$ whenever defined. 
Let us assume $R_{0} \geqslant 1 / \sqrt{2}$ (see the first lines" of this section; we can do without this assumption, taking $Q=\left\langle 0, R_{0} \sqrt{2}\right\rangle \times\left\langle 0, R_{0} \sqrt{2}\right\rangle$ instead of that above). Then any rectangle $S^{*} \subset Q$ can be represented as an image of $K$ by a contractive one-to-one mapping $\varphi$. So $S=\varphi^{i}\left(S^{*}\right)=\varphi^{i}(\varphi(K)) \in L$, since $\varphi^{i} \circ \varphi$ is a contractive one-to-one mapping. Put for $[u, v] \epsilon Q^{*}$

$$
\begin{aligned}
& f^{i}(u, v)=f\left(\varphi^{i}(u, v)\right) \quad \text { when proving (13.4), } \\
& f^{i}(u, v)=\left|x-\varphi^{i}(u, v)\right|^{-1} \text { when proving (13.5), }
\end{aligned}
$$

and denote by $\sigma^{i} \subset Q$ the projection of $\sigma \mid F_{i}$ on $Q$, defined by

$$
\int g(u, v) d \sigma^{i}=\int_{F_{i}} g(\psi(x)) d \sigma
$$

for all $g$ continuous in $Q$, where $\psi$ means the mapping inverse to $p^{i}$. Let us estimate $\int f^{i} d \sigma^{i}$.

The $h$-averaging of a measure $\tau$ in the $[u, v]$-plane consists in replacing $\tau$ by $\tau_{h}$ with

$$
\tau_{h}(E)=|R|^{-1} \int_{R} \tau(E+[p, q]) d p d q,
$$

where $\quad E+[p, q]=\{[\xi, \eta]:[\xi-p, \eta-q] \epsilon E\}, \quad R=\{[p, q]:|p| \leqslant h$, $|q| \leqslant h\},|R|=4 h^{2}, h>0$, and $d p d q$ denotes the integration with respect to (Lebesgue) area. Put, as in the theory of probability,

$$
\begin{aligned}
& t(u, v)=\tau\left(Q_{w v}\right), \quad Q_{u v}=(-\infty, u\rangle \times(-\infty, v\rangle, \\
& t_{h}(u, v) \stackrel{d f}{=}|R|^{-1} \int_{R} t(u+p, v+q) d p d q \\
& \quad=|R|^{-1} \iint_{R} \tau\left(Q_{u v}+[p, q]\right) d p d q=\tau_{h}\left(Q_{u v}\right) .
\end{aligned}
$$

Suppose now that $\tau$ has a finite absolute mass (see section 5). Then $t_{h}$, the $h$-average of $t$, is continuous, since $t$ is bounded (by a transformation as in (13.8)). Moreover,

$$
\sup \left|t_{h}\right| \leqslant \sup |t| .
$$

The averaging may be iterated, e.g.

$$
\begin{aligned}
t_{h h}(u, v) \stackrel{d t}{=}\left(t_{h}\right)_{h} & =|R|^{-1} \int_{-h}^{h} d p \int_{-h}^{h} t_{h}(u+p, v+q) d q \\
& =|R|^{-1} \int_{u=h}^{u+h} d p^{\prime} \int_{v-h}^{v+h} t_{h}\left(p^{\prime}, q^{\prime}\right) d q^{\prime}
\end{aligned}
$$

The last formula shows the existence of a continuous derivative $\partial^{2} t_{h h} / \partial u \partial v$, which is the density of $\left(\tau_{h}\right)_{h}$. Indeed,

$$
\int_{a}^{b} d u \int_{c}^{d}\left(\partial^{2} t_{h h} / \partial u \partial v\right) d v=\left(\tau_{h}\right)_{h}((a, b\rangle \times(c, d\rangle)
$$

(by (13.6) with $\tau_{h}$ on the place of $\tau$ ).

On the other hand, let $g(u, v) \in C_{0}\left(R^{2}\right)$. To any $\varepsilon>0$, choose a $\delta>0$ such that $\left|g(u, v)-g\left(u^{\prime}, v^{\prime}\right)\right|<\varepsilon$ provided $\left|u-u^{\prime}\right|<\delta, \quad\left|v-v^{\prime}\right|<\delta$. With $2 h<\delta$ we obtain $\left|\int g d\left(\tau_{h}\right)_{h}-\int g d \tau\right|<\varepsilon|\tau|(G)$, where $G$ is the compact set outside of which $g=0$. So, $\left(\tau_{h}\right)_{h} \rightarrow \tau(h \rightarrow 0)$ (see e. g. [13]).

Write $\sigma^{i n}=\left(\sigma_{1 / n}^{i}\right)_{1 / n}$ for $n$ satisfying $1 / n<r$; we allow from now on only such values for $n$. Then $\sigma^{i n} \subset Q^{*}$ vanishes near its boundary and $\sigma^{i n} \rightarrow \sigma^{i}$. Put

$$
s^{i n}(u, v)=\sigma^{i n}\left(Q_{u v}\right), \quad s^{i}(u, v)=\sigma^{i}\left(Q_{u v}\right)
$$

and observe that

(13.9) $\quad \sup _{Q^{*}}\left|s^{i}\right|=\sup \left|\sigma^{i}\left(S^{*}\right)\right| \leqslant[\sigma], \quad s^{i n} \epsilon C^{2}(Q), d \sigma^{i n}=s_{u v}^{i n} d u d v$,

(the second upper bound is taken for all rectangles $S^{*} \subset Q ; u, v$ as indices denote differentiation). The inequality results from the fact that $\varphi^{i}\left(S^{*}\right) \in L$.

For $u$ or $v$ near to $-r$ we have $s^{i n}=0$, so integration by parts yields

$$
\begin{aligned}
\iint_{Q^{*}} s f_{u v}^{i n} d u d v=f s^{i n}(1+r & 1+r)-\int_{-r}^{1+r} f_{u} s^{i n}(u, 1+r) d u- \\
& -\int_{-r}^{1+r} f_{v} s^{i n}(1+r, v) d v+\iint_{Q^{*}} f_{u v} s^{i n}(u, v) d u d v
\end{aligned}
$$

$\left(f_{u v} s^{i n}(u, v)\right.$ means $f_{u v}(u, v) s^{i n}(u, v)$ and so on). Thus with

(13.10) $\quad M_{i}=|f(1+r, 1+r)|+\int_{-r}^{1+r}\left|f_{u}(u, 1+r)\right| d u+$

$$
+\int_{-r}^{1+r}|f(1+r, v)| d v+\iint_{Q}\left|f_{u v}\right| d u d v
$$

we have

$$
\left|\int f^{i} d \sigma^{i n}\right| \leqslant M_{i} \max _{Q^{*}}\left|s^{i n}\right| \leqslant M_{i} \max _{Q^{*}}\left|s^{i}\right| \leqslant M_{i}[\sigma]
$$

by applying (13.7) twice to obtain the second inequality, and then (13.9) for the last one.

Letting $n \rightarrow \infty$ we see that

$$
\left|\int f^{i} d \sigma^{i}\right| \leqslant M_{i}[\sigma]
$$


So our lemma is established with $M=\sum_{i=1}^{N} M_{i}$, which is easily seen (in the case of proving (13.5)) to depend on $x$ in a continuous way.

Our proof gives rise to a new definition of the total variation (which meets Hardy's definition of a function of bounded variation, see e.g. [10]) establishing a duality between it and a somewhat modified Tchebycheff's norm. We will devote another paper to consider this in detail.

\section{EQUILIBRIUM THEORY}

14. We shall now be concerned with the class of measures

$$
K(\nu, m)=K(\nu) \frown\{\sigma:[\sigma] \geqslant m, \sigma(F)=0\} .
$$

(see (11.1), (13.3), (13.1)). It is vaguely compact, the last set being closed in $K(\nu)$ and $K(\nu)$ compact; we suppose $0<m<\sup \left\{\min \left(\nu(S), \nu\left(S^{\prime}\right)\right)\right.$ : $S \in L\}$, so $K(\nu, m)$ is non-void. By Lemma 2 we have at once

THEOREM I. There is a minimal measure $\alpha \in K(\nu, m)$ :

$$
\|\alpha\|^{2} \leqslant\|\sigma\|^{2} \quad(\sigma \in K(\nu, m)) .
$$

Let us study now to what extent $\alpha$ has the familiar properties of classical equilibrium distribution.

The minimal measure may not be unique. In what follows we mean by $\alpha$ any concrete one.

THEOREM II (neutralization theorem). There is either an $S_{+}=S_{+}(\alpha) \in I$ such that

(14.1) $\alpha\left(S_{+}\right)=[\alpha]=m$ and $\alpha \geqslant 0$ on $S_{+}, \alpha \leqslant 0$ on $S_{-}=\left(S_{+}\right)^{\prime}$,

or an $S_{+}=S_{+}(-\alpha)$ such that (14.1) is satisfied by $-\alpha$.

By $a \geqslant 0$ on $S_{+}$we mean $a \mid S_{+} \geqslant 0$.

Proof. There is, by Lemma 6 , an $S_{+} \in I$ with $\left|\alpha\left(S_{+}\right)\right|=[a]$. Suppose $\alpha\left(S_{+}\right)>0$, and, this being so, set $S_{+}=S_{+}(\alpha)$ (in the case of $-\alpha\left(S_{+}\right)>$ $>0$ set $S_{+}=S_{+}(-\alpha)$ and substitute the minimal measure $-\alpha$ for $\alpha$ in what follows). $[\alpha]=m$, otherwise $m[\alpha]^{-1} \alpha \in K(\nu, m)$ while its energy is $m^{2}[\alpha]^{-2}\|\alpha\|^{2}<\|\alpha\|^{2}$.

Let $K^{*}=K(v, m) \cap\left\{\sigma: \sigma \geqslant 0\right.$ on $S_{+}, \sigma \leqslant 0$ on $\left.S_{-}\right\}$be the (non-void) class of measures already satisfying the inequalities (14.1). It is compact by the argument given above. Let $\beta \in K^{*}$ be minimal: $\|\beta\|^{2} \leqslant\|\sigma\|^{2}\left(\sigma \in K^{*}\right)$. Now, $U^{\beta}$ being continuous in the whole space (Lemma 1) and regular at the infinity (see section 8 ), we have

$$
-\infty<v \stackrel{d f}{=} \inf _{x} U^{\beta}(x)<0<\sup _{x} U^{\beta}(x) \stackrel{d f}{=} V<\infty .
$$

We use the following theorem, postponing its demonstration to the section 15:

THEOREM III (saturation theorem). We have

$$
U^{\beta}(x)=V \quad\left(x \in S_{+}-E_{+}\right), \quad U^{\beta}(x)=v \quad\left(x \in S_{-}-E_{-}\right),
$$

the exceptional sets being saturated:

$$
\begin{aligned}
& \quad \beta\left(E_{ \pm}\right)= \pm \nu\left(E_{ \pm}\right) . \\
& \text {Put } \alpha=\beta+\tau \text {, then } \\
& \tau \leqslant 0 \text { on } E_{+}, \quad \tau \geqslant 0 \text { on } E_{-}, \quad \tau\left(S_{+}\right)=\tau\left(S_{-}\right)=0
\end{aligned}
$$

and the integrals of $U^{\beta} d \tau$ satisfy the inequalities:

$$
\begin{array}{ll}
\int_{E_{+}} \geqslant V \tau\left(E_{+}\right), & \int_{S_{+}-E_{+}}=V \tau\left(S_{+}-E_{+}\right), \\
\int_{E_{-}} \geqslant v \tau\left(E_{-}\right), & \int_{S_{-} E_{-}}=v \tau\left(S_{-}-E_{-}\right) .
\end{array}
$$

Summing up we get $(\beta, \tau) \geqslant 0$ (see $(9.1)$ ), so

$$
\|\alpha\|^{2}=\dot{\|} \beta\left\|^{2}+2(\beta, \tau)+\right\| \tau\left\|^{2} \geqslant\right\| \beta \|^{2}
$$

with equality only when $\tau \equiv 0$. But this is just the case, since $K^{*} \subset K(\nu, m)$ implies $\|\alpha\|^{2} \leqslant\|\beta\|^{2}$. So $\alpha \equiv \beta$ and the proof of (14.1) is achieved.

15. We proceed now to prove theorem III.

$E_{+}$and $E_{-}$are $\nu$ - and $\beta$-measurable as intersections of $S_{+}$with open sets. Suppose $\nu\left(E_{+}\right)-\beta\left(E_{+}\right)>0$ and put $E_{\varepsilon}=\left\{x: x \in\left(S_{+}\right)^{-}, U^{\beta}(x) \leqslant V-\varepsilon\right\}$; this is a compact set. With some $\varepsilon \epsilon(0, V)$, we have $a=\nu\left(E_{\varepsilon}\right)-\beta\left(E_{\varepsilon}\right)>0$. Secondly, $b=\beta\left(S_{+-}-E_{\varepsilon}\right)>0$ - if not, $U^{\beta}$ would be subharmonic in $R^{3}-E_{s}$, continuous in $R^{3}$, regular harmonic at the infinity, and $\leqslant V-\varepsilon$ on $E_{s}$, thus everywhere - a contradiction with (14.2). Define now $\tau \subset S_{+}$ by

Then

$$
\begin{aligned}
& d \tau(x)=a d \beta(x) \geqslant 0 \quad\left(x \in S_{+}-E_{\varepsilon}\right) \\
& d \tau(x)=-b d(\nu-\beta)(x) \leqslant 0 \quad\left(x \in E_{\varepsilon}\right) .
\end{aligned}
$$

$$
\begin{aligned}
(\beta, \tau) & =\int_{E_{e}} U^{\beta} d \tau+\int_{S_{+}-E_{\varepsilon}} U^{\beta} d \tau \\
& >(V-\varepsilon) \tau\left(E_{\varepsilon}\right)+(V-\varepsilon) \tau\left(S_{+}-E_{s}\right)=0,
\end{aligned}
$$

since $\tau\left(S_{+}\right)=0$. The strong inequality results from the fact that $S_{+}-E_{\varepsilon / 2}$ contains a positive $\beta$ - (and $\tau$-) mass, by the same reasoning as above. For small $t>0$, we have $\beta-t \tau \epsilon K^{*}$ and

$$
\|\beta-t \tau\|^{2}=\|\beta\|^{2}-2 t(\beta, \tau)+t^{2}\|\tau\|^{2}<\|\beta\|^{2},
$$


contrary to the minimal character of $\beta$. The second part of the theorem is proved in a symmetrical way.

16. THEOREM IV (filling up theorem). The support of $\alpha=\beta$ is the whole support of $\nu$.

Proof. We show e. g. that the support of $\beta^{+}$contains that of $\nu \mid S_{+}$. Otherwise, there would be an $x_{0} \epsilon S_{+}$on the support of $v \mid S_{+}$but not on that of $\beta^{+}$, and consequently a small open ball $B_{0}$ with centre $x_{0}$ and rectifiable $\partial B_{0} \cap F$ such that $\beta^{+}(B)=0, \nu(B)>0\left(B=B_{0} \cap S_{+}-\partial S_{+}\right)$ (the cancelling of $\partial S_{+}$is legitimate, since $v\left(\partial S_{+}\right)=0$ - see Lemma 3$)$. $B$ lies then in the open region of harmonicity of $U^{\beta}$. By Lemma 3 , we can fix a $\delta>0$ with $v(\delta \partial B)<\frac{1}{2} v(B)$. Then, by the harmonicity mentioned, $2 \varepsilon=V-\sup \left\{U^{\beta}(x): x \in B-\delta \partial B\right\}>0$. We have $a=\nu(B-\delta \partial B)>0$ and $b=\beta\left(S_{+}-E_{s}\right)>0$, where $E_{s}$ is defined and the inequality established as in section 15 Put

$d \tau(x)=a d \beta(x) \geqslant 0\left(x \in S_{+}-E_{s}\right), \quad d \tau(x)=-b d v(x) \leqslant 0 \quad(x \in B-\delta \partial B)$, for other $x, d \tau(x)=0$. From now on the proof runs like the preceding one.

THEOREM $V$ (uniqueness theorem). Two minimal measures in $K(\nu, m)$ coincide provided the supports of their positive (or negative) parts coincide.

Proof. Let $\alpha_{1}, \alpha_{2}$ be two minimal measures in $K(\nu, m)$ and the supports of $\alpha_{1}^{+}, \alpha_{2}^{+}$be identical. By Theorem IV, $S_{+}\left(\alpha_{1}\right)=$ (support of $\left.\alpha_{1}^{+}\right) \cup \mathscr{N}=$ (support of $\left.\alpha_{2}^{+}\right) \cup \mathscr{N}$, where $\mathscr{N} \subset F-$ (support of $v$ ). Hence $\alpha_{2} \geqslant 0$ on $S_{+}\left(\alpha_{1}\right)$ and $\alpha_{2}\left(S_{+}\left(\alpha_{1}\right)\right)=\alpha_{2}^{+}(F)=m$, the last equation being valid by Theorem I for any minimal measure. So we can put $S_{+}\left(a_{2}\right)$ $=S_{+}\left(\alpha_{1}\right)$ and substitute $\beta=\alpha_{1}, \alpha=\alpha_{2}$ in the last part of section 14 .

Generally, there are many minimal measures in $K(v, m)$. E. g., $F$ being a sphere and $\nu=$ const. $\times$ area, each minimal measure produces other ones by rotations. We put the following problem, which seems rather difficult (contrary to that from section 13):

P 451. Characterize the pairs $(F, v)$ such that, for sufficiently small $m$, there exists only one minimal measure in $K(v, m)$ - or, show that no such $F$ and $\nu$ exist.

\section{ESTIMATION OF $[\sigma]$ IN TERMS OF $\|\sigma\|$}

17. Our task is now to estimate the energy $\|\sigma\|^{2}$ from below in $K(\nu, m)$, i. e. in $K(v)$ in terms of [ $\sigma$ ]. All constants below depend on $F$ only, excepting $m_{0}$ which depends also on $\nu$. Define the "inverse function" to (12.2) by

$$
\omega^{-1}\left(\varepsilon ; \nu, l_{0}\right)=\sup \left\{\delta: \omega\left(\delta ; \nu, l_{0}\right) \leqslant \varepsilon\right\} \quad(\varepsilon>0) .
$$

$\omega^{-1}$ goes to 0 with $\varepsilon$ (otherwise $v=0$ in each small set which implies $\nu \equiv 0)$, so there is an $m_{0}>0$ such that $4 \delta \stackrel{\text { df }}{=} \omega^{-1}\left(\frac{1}{2} m ; \nu, l_{0}\right) \leqslant \delta_{0}$ for $0<m \leqslant m_{0}$ (see section 10). We fix such an $m$ and the corresponding $\delta$ and prepare a finite partition of $F$ in disjoint sets $F_{1}, \ldots, F_{N_{0}}$ each contained in a ball $B_{i}=\left\{x:\left|x-x_{i}\right| \leqslant \delta\right\}\left(x_{i} \in F_{i}\right)$. The number $N_{0}$ depends on $\delta$, but it is easily seen that we can always have $N_{0} \leqslant c_{3} / \delta^{2}$ with constant $c_{3}$ (a proof can be based on partitions of $Q$ as in the proof of Lemma 7).

Let $\alpha$ be a fixed minimal measure in $K(v, m)$. We change the order of the $F_{i}$ 's so as to have, with some $n \leqslant N_{0}$

$$
S_{+}=S_{+}(\alpha)=A \cup \bigcup_{i=1}^{n} F_{i}^{*}, \quad A=\left(S_{+}\right)^{-} \cap(4 \delta) C S_{+}, \quad F_{i}^{*}=F_{i}-A
$$

(see (12.1)).

Put (see section 4)

$$
\alpha_{*}=\alpha\left|A \geqslant 0, \alpha_{i}=\alpha\right| F_{i}^{*} \geqslant 0 \quad(i=1, \ldots, n) .
$$

The idea of our present consideration is the following. The danger that $\|\alpha\|^{2}$ will be small consists in the tendency of positive masses to draw near the negatives (and vice versa), which makes $\alpha_{*}$ relatively large. Controlling this phenomenon is just the object of $\nu$-majoration. In fact, this enables us, after separating the contributions of each $\alpha_{i}$, to neglect that of $\alpha_{*}$ - thanks to $\delta$ being so small as to cause $\alpha_{*}(A) \leqslant \frac{1}{2}[\alpha]$. Then we may estimate the contributions of $\alpha_{1}, \ldots, \alpha_{n}$ thanks to their distance from negative masses being bounded away from 0 . For concrete $F$, this could often be done by direct estimation of the integral. We have chosen an easier way. It seems somewhat prodigal, but the example of a spherical condenser shows the order of the estimate for $m \rightarrow 0$ to be sharp.

In fact, let $G$ be the unit sphere, $H$ the concentric sphere of radius $1+4 \delta, \sigma^{+} \subset G, \sigma^{-} \subset H$ with $\sigma^{+}(G)=\sigma^{-}(H)=m$. Then we have the sharp estimation $\|\sigma\|^{2} \geqslant m^{2}\left(1-(1+4 \delta)^{-1}\right) \approx 4 \delta m^{2}$ (obtained as in the last part of the proof we begin), while by partition of $G$ analogous to the above one and by the method of our proof we obtain $\|\sigma\|^{2} \geqslant$ const. $\delta m^{2}$.

Let us form a surface $P=\partial E, E=\left\{x: x \in R^{3}, \varrho\left(x, S_{+}-A\right)<4 \delta\right\}$. Observe that $\partial S_{+} \cap E=0$. We do not prove that $P$ is really a surface, since we shall use only $1^{\circ}$ its (obvious) compactness, $2^{\circ}$ its property of separating $F_{1}^{*}, \ldots, F_{n}^{*}$ from $S_{-}$. Let us prove the last property. If it would not hold good, then an $x_{-} \epsilon S_{-}$ougth to lie in $E$ and thus in some ball $B=\left\{x:\left|x-x_{*}\right|<4 \delta\right\}, x_{*} \in S_{+}-A$. Now, $4 \delta \leqslant \delta_{0}$, hence $F \cap B$ is connected (see section 10) and $x_{-}, x_{*}$ can be joined in $F \cap B \subset F \cap E$ by an are $J$. But $J$ must then cut $\partial S_{+}$, contrary to $J \subset E$. 
Put else $\alpha_{0}=\alpha_{*} \mid A \cap \mathbb{E}^{-}$. To each $\alpha_{i}$ there exists ([5], $\mathrm{n}^{\text {os }} 8-10$ ) a positive measure $\beta_{i} \subset P^{*}=P \cup(F-E)$ of finite energy with the following properties:

$$
\beta_{i}\left(P^{*}\right)=m_{i} \overline{\overline{\mathrm{df}}} \alpha_{i}\left(\mathbb{S}_{+}\right),
$$

$$
\left\|\alpha_{i}-\beta_{i}\right\| \leqslant\left\|\alpha_{i}-\tau\right\| \text { for all } \tau \subset P^{*} \text { with } \tau\left(P^{*}\right)=m_{i},
$$

(17.4) $\quad U^{\beta_{i}}(x) \leqslant U^{\alpha_{i}}(x) \quad\left(x \in R^{3}\right), \quad U^{\beta_{i}}(x)=U^{\alpha_{i}}(x) \quad\left(x \in P^{*}-P_{i}\right)$,

$P_{i}$ being a set of interior capacity null, i. e. negligible for every measure of finite energy - in particular $\beta_{k}\left(P_{i}\right)=0(i, k=0,1, \ldots, n)$. We say that $\beta_{i}$ is obtained by balayage of $\alpha_{i}$ onto $P^{*}$. Balayage is a linear operation; this appears from the condition (17.4) which characterizes the balayed measure completely ([5], $\mathrm{n}^{0} 12$ ).

We prove (17.2), since the reader will not find this well-known equation explicitly in [5]. Let $\mu$ be the capacitary distribution (see after $(19.2))$ on $K=E \frown P^{*}$. Then $\beta_{i}(K)=\int_{K} 1 d \beta_{i}=\int U^{\mu} d \beta_{i}=\left(\beta_{i}, \mu\right)=\left(\alpha_{i}, \mu\right)$ $=\alpha_{i}(K)$, since $\mu \mid E \equiv 0$ and (17.4) holds.

Now, (17.4) implies

$$
\left(\alpha_{i}-\beta_{i}, \alpha_{k}-\beta_{k}\right)=\int_{S_{+}} U^{\alpha_{i}-\beta_{i}} d \alpha_{k}-\int_{P^{*}-P_{i}} U^{\alpha_{i}-\beta_{i}} d \beta_{l_{c}} \geqslant 0 .
$$

Put $\alpha_{R}=\alpha \mid S_{+} \cap E^{-}=\sum_{i=0}^{n} \alpha_{i}$. The balayage of $\alpha_{R}$ onto $P^{*}$ gives a measure $\beta=\sum_{i=0}^{n} \beta_{i}$. By $(17.2-3), \beta\left(P^{*}\right)=\alpha_{R}\left(S_{+} \cap E^{-}\right)$and $\left\|\beta-\alpha_{R}\right\|$ $\leqslant\left\|\tau-\alpha_{R}\right\|$ for all $\tau \subset P^{*}$ with $\tau\left(P^{*}\right)=\alpha_{R}\left(S_{+} \cap E^{-}\right)$. Then $\tau=\alpha_{R}-\alpha=$ $=-\alpha \mid F-E$ is an admissible measure and we have, by (17.3-5),

$$
\begin{aligned}
\|\alpha\|^{2} & =\left\|\alpha_{R}-\left(\alpha_{R}-\alpha\right)\right\|^{2} \geqslant\left\|\alpha_{R}-\beta\right\|^{2}=\left\|\sum_{i=0}^{n}\left(\alpha_{i}-\beta_{i}\right)\right\|^{2} \\
& =\sum_{i, k=0}^{n}\left(\alpha_{i}-\beta_{i}, \alpha_{k}-\beta_{k}\right) \geqslant \sum_{i=k} \geqslant \sum_{i=1}^{n}\left\|\alpha_{i}-\beta_{i}\right\|^{2}
\end{aligned}
$$

(just in the last sum $\alpha_{*}$ is definitively eliminated).

We estimate now $\left\|\alpha_{i}-\beta_{i}\right\|^{2}$. Put $K_{+}=\partial B_{i}$ (defined at the beginning of this section), $K_{-}=\partial B_{i}^{*}, B_{i}^{*}=\left\{x:\left|x-x_{i}\right| \geqslant 4 \delta\right\}$. Let $\sigma^{+} \subset K_{+}, \sigma^{-} \subset K$ be obtained by balaying the mass $m_{i}$ from the centre $x_{i}$, i. $\theta$. let these measures be of constant density with respect to area, each of mass $m_{i}$. With $\sigma=\sigma^{+}-\sigma^{-}$we have $U^{\sigma}=m_{i}\left(\delta^{-1}-(4 \delta)^{-1}\right)=3 m_{i} / 4 \delta$ in $B_{i}$ and $U^{o}=0$ in $B_{i}^{*}$. On the other hand, $\alpha_{i}-\sigma^{+} \subset B_{i}^{-}, \sigma^{-}-\beta_{i} \subset B_{i}^{*-}$, whereas

their total masses are 0 , whence the integrals of $U^{\sigma}$ with respect to those measures vanish:

$$
\begin{aligned}
\left\|\alpha_{i}-\beta_{i}\right\|^{2} & =\left\|\sigma-\left(\sigma^{+}-\alpha_{i}+\beta_{i}-\sigma^{-}\right)\right\|^{2} \\
& =\|\sigma\|^{2}-2 \int U^{\sigma} d\left\{\left(\sigma^{+}-\alpha_{i}\right)+\left(\beta_{i}-\sigma^{-}\right)\right\}+\left\|\sigma-\left(\alpha_{i}-\beta_{i}\right)\right\|^{2} \\
& \geqslant\|\sigma\|^{2}=\frac{3}{4 \delta} m_{i}^{2} .
\end{aligned}
$$

Putting this together with (17.6) we obtain

$$
\|\alpha\|^{2} \geqslant \sum_{i=1}^{n} \frac{3}{4 \delta} m_{i}^{2} \geqslant \frac{3}{4 \delta} n\left(\frac{m}{2 n}\right)^{2} \geqslant c_{4} \cdot 4 \delta \cdot m^{2}
$$

because of $\sum_{1}^{n} m_{i} \geqslant \frac{1}{2} m, m_{i} \geqslant 0$, and of $n \leqslant N_{0} \leqslant c_{3} / \delta^{2}$ (see the initial part of this proof). In view of $\alpha$ being minimal in $K(v, m)$ we have the following

THEOREM VI. Let $F$ and $v$ satisfy the conditions of sections 10 and 11. Then for any $\sigma \in K(\nu)$ with $[\sigma] \leqslant m_{0}$ and $\sigma(F)=0$ we have

$$
\|\sigma\|^{2} \geqslant c_{4}[\sigma]^{2} \omega^{-1}\left(\frac{1}{2}[\sigma] ; \nu, l_{0}\right),
$$

where $o_{4}>0$ and $l_{0}$ depend on $F$ but not on $v$ (nor $\left.\sigma\right)$, and $m_{0}$ depends on $F$ and $\nu$.

Remarks. Our considerations were done with $m=[a] \leqslant m_{0}$, but if $m>m_{0}, K(\nu, m) \subset K\left(\nu, m_{0}\right)$, so with a larger $c_{4}^{\prime}=\frac{1}{2} c_{4}^{\prime} \nu\left(F^{\prime}\right) m_{0}^{-1}$ (now depending on $v$ ) (17.7) holds in the whole $K(\nu)$.

The condition $F \epsilon C^{2}$ may be weakened. $F$ need not be a connected surface - it could be a finite union of disjoint $C^{2}$ surfaces as well. We may even allow these surfaces to intersect, but they must not be mutually tangent. Precisely, we allow $F=\bigcup_{i=1}^{k} P_{i}$, where $1^{0}$ each $P_{i}$ is a compact surface element of class $O^{2}, 2^{\circ}$ the angle formed by $P_{i}$ and $P_{k}(i \neq k)$ at each common point lies between $\theta$ and $\pi-\theta, \theta$ being a fixed positive number. Some additional care is then needed, especially in proving Theorem VI, nevertheless all theorems excepting IV (which fails if a $P_{i}$ surrounds another) are valid on such an $F$.

18. To elucidate the influence of $\omega^{-1}$ on (17.7) let us examine the case of an $\nu$ of bounded density: $\nu(E) \leqslant g^{*}|E|$ for Borel sets $E \subset F$, or the more general one of an $v$ satisfying with some $a \in(0,1), g \in(0, \infty)$ the inequality

$$
\omega\left(\delta ; \nu, l_{0}\right) \leqslant g \delta^{a} \quad\left(\delta \leqslant \delta_{0}\right) .
$$

Colloquium Mathematicum XI. 2 
The former case is that of $a=1$, since $|\delta C| \leqslant c_{5} \delta \quad\left(c_{5}=\right.$ positive constant), if the length $|C| \leqslant l_{0}$. To see this, divide $C$ by points $x_{0}, x_{1}, \ldots$ $\ldots, x_{k}=x_{0}$ into $k$ ares of lengths $\leqslant 2 \delta$; to this purpose, $k=\left[l_{0} / 2 \delta\right]+1$ is sufficient. When taking an $x \in \delta C$, going to the nearest point $x^{\prime} \in C$ and from $x^{\prime}$ to the nearest $x_{i}$, we see that $\delta C \subset \bigcup_{i=1}^{k} F\left(x_{i}, 2 \delta\right)$, thus $|\delta C| \leqslant c_{5} \delta$, $c_{5}=\pi c_{2} \cdot 4 \delta \leqslant 6 c_{2} l_{0}$ for $\delta \leqslant l_{0}$ (consult sections 10 and 12 for notations and for eliminating, if desired, the limitation on $\delta$ ). (18.1) implies

$$
\omega^{-1}\left(\varepsilon ; \nu, l_{0}\right) \geqslant g^{-1 / a} \varepsilon^{1 / a}
$$

thus (17.7) becomes: $\|\sigma\|^{2} \geqslant c_{6}[\sigma]^{2+1 / a}, c_{6}=c_{4}(2 g)^{-1 / a}$. When $a=1$, we get a particularly simple

THEOREM VII. If $F$ satisfies the conditions either of section 10 or of 17 and $0 \leqslant v(E) \leqslant g|E|$, where $|E|$ is the area of an arbitrary Borel set $E \subset F$ and $v$ a measure supported by $F$, then

$$
[\sigma]^{3} \leqslant c^{*} g\|\sigma\|^{2} \quad\left(c^{*}=2 c_{5} / c_{4}\right)
$$

for any $\sigma \in K(\nu)$ with $\|\sigma\|^{2} \leqslant b=m_{0}^{3} c_{4} / 2 g c_{5}$ and $\sigma(F)=0$. The constants $c_{4}, c_{5}>0$ depend on $F$ only; $m_{0}$ can be put equal to $2 c_{5} g \delta_{0}$ (see section 10 ).

To complete the above proof observe that $1^{0}$ if $\nu^{*}(E) \equiv g|E|$ then its potential is continuous [8] and, by Lemma 1 , so is that of $\nu ; 2^{\circ}$ if $\|\sigma\|^{2} \leqslant b$, $\sigma \epsilon K(\nu)$ and $[\sigma]=m>m_{0}$, then $\sigma^{*}=m_{0} m^{-1} \sigma \epsilon K\left(\nu, m_{0}\right)$, whence - by Theorem VI $-\left\|m_{0} m^{-1} \sigma\right\|^{2} \geqslant c_{4} m_{0}^{2} m_{0} / 2 g c_{5}$ and $\|\sigma\|^{2}>b$; thus $\|\sigma\|^{2} \leqslant b$ implies $[\sigma] \leqslant m_{0}$ in the present circumstances.

19. An explicit theorem is also available in general circumstances. It depends on the following

LEMMA 8. $\operatorname{cap} \delta \sigma \leqslant c_{8}^{2} / \log 1 / \delta \quad\left(|C| \leqslant l_{0}, \delta \leqslant \delta^{*}\right)$, where $c_{8}$ and $\delta^{*}>0$ depend on $F$ and $l_{0}$ only (see (12.1)).

Proof. Represent $C$ as a contractive image of the circle $K=\left\{\left[u_{1}, u_{2}\right]\right.$ : $\left.u_{1}^{2}+u_{2}^{2}=R_{0}^{2}\right\}$ of the $\left[u_{1}, u_{2}\right]=u$-plane $\left(R_{0}=l_{0} / 2 \pi\right)$, i. e. $O: x=\varphi(u)$ with $\left|\varphi(u)-\varphi\left(u^{*}\right)\right| \leqslant\left|u-u^{*}\right|\left(u, u^{*} \epsilon K\right)$. Define the "centres" $x^{i}$ of sets $F^{i}=F\left(x^{i}, \delta\right)=F \cap\left\{x:\left|x-x^{i}\right| \leqslant \delta\right\}$ by induction as follows. Take any $x^{1} \in C$. If $F^{1}, \ldots, F^{k}$ are defined, then choose any $x_{k+1} \epsilon O-F^{1}-\ldots-F^{k}$ if such a point exists; otherwise the construction is ended, and this must be the case for a finite $k=k^{\prime}-$ to see this, cover $R^{3}$ with cubes of diameter $\leqslant \delta$ and observe that none contains two points $x^{i}, x^{k}(i \neq k)$ of our sequence. Put $E^{1}=F^{\prime}\left(x^{1}, 2 \delta\right), E_{k}=F^{\prime}\left(x^{l}, 2 \delta\right)-E^{k-1}-\ldots-E^{1}$. We have

$$
\left|x^{i}-x^{k}\right| \geqslant \delta \quad\left(i<k=2, \ldots, k^{\prime}\right), \quad \delta C \subset \bigcup_{k=1}^{k^{\prime}} E^{k}
$$

Let $x^{i}=\varphi\left(u^{i}\right)$. Then $\left|u^{i}-u^{k}\right| \geqslant \delta$. Take a circle in the $u_{1}, u_{2}$-plane: $\left\{u:|u|=9 R_{0}\right\}$, and transport each $F\left(x^{k}, 2 \delta\right.$ ) (so each $E^{k}$ ) by a rigid motion - denoting it by $*$ - into the $u_{1}, u_{2}, u_{3}$-space so as $x_{*}^{k}=9 u^{k}$ and $F^{\prime}\left(x^{k}, 2 \delta\right)_{*}$ becomes tangent to the $u_{1}, u_{2}$-plane at this point. Consider two points $y^{i} \in E^{i}, y^{k} \in E^{k}$. If $i=k$, their distance is unaltered. If $i \neq k$ their distance before moving was $\left|y^{i}-y^{k}\right| \leqslant r^{i k}+4 \delta \quad\left(r^{i k}=\left|x^{i}-x^{k}\right|\right.$ $\left.\leqslant\left|u^{i}-u^{k}\right|\right)$; now, in the $u$-space, $\left|y_{*}^{i}-y_{*}^{k}\right| \geqslant\left|9 u^{i}-9 u^{k}\right|-4 \delta \geqslant 9 r^{i k}-4 \delta$ $\geqslant r^{i k}+4 \delta$ by (19.1). So all distances are increased, whence

$$
\operatorname{cap} \delta C \leqslant \operatorname{cap} \cup E^{k} \leqslant \operatorname{cap} \cup E_{*}^{k} .
$$

Let $H^{k}$ be the orthogonal projection of $E_{*}^{k}$ on the $u_{1}, u_{2}$-plane. If $\delta^{*}$ was taken small enough, the angle between the last and any tangent plane to $E_{*}^{k}$ is $\leqslant \pi / 3$, so

$$
\operatorname{cap} \cup E_{*}^{k} \leqslant 2 \operatorname{cap} \bigcup H^{k} \leqslant 2 \operatorname{cap} R,
$$

where $R \supset \cup B^{k}$ is the plane annulus $9 R_{0}-2 \delta \leqslant|u| \leqslant 9 R_{0}+2 \delta$. Observe that $R$ is a contractive image of the strip $T^{2}=\langle 0,4 \delta\rangle \times\langle 0, l\rangle(l=2 \pi(9+$ $+2 \delta)$ ), whence $\operatorname{cap} R \leqslant \operatorname{cap} T^{2}\left({ }^{4}\right)$

Let $\tau_{2} \subset T^{2}, d \tau_{2}=d u_{1} d u_{2}$ in $T^{2} . U^{\tau_{2}}$ attains obviously its minimum on $T^{2}$ at $(0,0)$. Thus, we have for $u \epsilon T^{2}$

$$
\begin{aligned}
U^{\tau_{2}}(u) & \geqslant U^{\tau_{2}}(0,0)=\int_{0}^{4 \delta} d u_{1} \int_{0}^{l}\left(u_{1}^{2}+u_{2}^{2}\right)^{-1 / 2} d u_{2} \\
& =\int_{0}^{4 \delta}\left\{\log \left|l+\left(l^{2}+u_{1}^{2}\right)^{1 / 2}\right|-\log u_{1}\right\} d u_{1} \\
& \geqslant 4 \delta \log 2 l-4 \delta(\log 4 \delta+1)=a>0 \text { for small } \delta^{*} .
\end{aligned}
$$

Let $\mu$ be the capacitary distribution on $T^{2}$, i. e. $\mu \subset T^{2}, U^{\mu} \equiv 1$ on $T^{2}$; then [4]

$$
\begin{aligned}
\operatorname{cap} T^{2} & =\mu\left(T^{2}\right)=\int 1 d \mu \leqslant \int U^{r_{2} / a} d \mu=\int U^{\mu} d \tau_{2} / a \\
& =\tau_{2}\left(T^{2}\right) / a=4 \delta l / a \geqslant A^{2} / \log 1 / \delta,
\end{aligned}
$$

with constant $A$ and $\delta \leqslant \delta^{*}$ ( $\delta^{*}$ suitably chosen). So our lemma is proved.

Remark. Observe that with $T^{*}=\langle-4 \delta, 4 \delta\rangle \times\langle-l, l\rangle$ and $\tau^{*} \subset T^{*}$ $d \tau^{*}=d u_{1} d u_{2}$ in $T^{*}, \max U^{\tau_{2}} \leqslant \max U^{*^{*}} \leqslant 4 U^{\tau_{2}}(0,0)$, and estimating

$\left.{ }^{4}\right)$ One could pass from $E^{k}$ directly to $T^{2}$, but we think the reader will appreciate the above way when generalizing in section 20. 
in (19.2) from above, we obtain $\operatorname{cap} T \geqslant B / \log 1 / \delta$. In this way one can show that $\sup \left\{\operatorname{cap} \delta C: C \subset F,|C| \leqslant l_{0}\right\} \geqslant B^{\prime} / \log 1 / \delta$. So our lemma is qualitatively sharp.

Now, $\nu(\delta C)^{2} \leqslant(\operatorname{cap} \delta C)\|\nu\|^{2}$, so for $\delta \leqslant \delta^{*}$ and correspondingly $\varepsilon \leqslant \varepsilon^{*}$,

$$
\omega\left(\delta ; \nu, l_{0}\right) \leqslant c_{8} / \sqrt{\log 1 / \delta}, \quad \omega^{-1}\left(\varepsilon ; \nu, l_{0}\right) \geqslant \exp -c_{8} / \varepsilon^{2} .
$$

So (17.7) becomes

$$
\|\sigma\|^{2} \geqslant c_{4}[\sigma]^{2} \exp -4 c_{8} /[\sigma]^{2} \geqslant c_{9} \exp -c_{10} /[\sigma]^{2} \quad\left([\sigma]^{2} \leqslant c_{11}\right)
$$

and we have

THEOREM VIII. Under conditions of Theorem VI,

$$
[\sigma]^{2} \leqslant \frac{c_{10}}{\log c_{9}\|\sigma\|^{-2}} \quad \text { if } \quad \sigma \epsilon K(v),\|\sigma\| \leqslant c_{12}
$$

where the positive constants $c_{9}, c_{10}, c_{12}$ depend (only) on $F$ and $v$.

To complete the proof, the argument used after Theorem VI under $2^{\circ}$ is to be adapted.

\section{GENERALIZATION TO HIGHER DIMENSIONS}

20. We have already mentioned that our results generalize to $N \geqslant 4$. Let us indicate how to adapt our considerations to this case. $F$ is then assumed to be an $N$-1-dimensional triangulable compact manifold of class $C^{N-1}$. In (12.2) $C$ is to be understood as a contractive image of the $N$-2-dimensional boundary of the $N$-1-dimensional ball $K$ of radius $R$, and in section $13 K$ should be this ball itself with $R=R_{0}$. In the proof of Lemma $7, Q$ will be an $N$-1-dimensional unit cube in the $u^{1}, u^{2}, \ldots, u^{N-1}$. -space; $d \sigma^{i n}=\left(\partial^{N-1} s^{i n} / \partial u^{1} \partial u^{2} \ldots \partial u^{N-1}\right) d u^{1} d u^{2} \ldots d u^{N-1}$, so we have to estimate $\int_{Q} f s^{i n} u^{1} u^{2} \ldots u^{N-1} d u^{1} d u^{2} \ldots d u^{N-1}$. It is to be written as an iterated integral and transformed by repeated integration by parts into a sum of $N-1, N-2, \ldots, 1$ - and 0 -dimensional integrals of products $s \cdot$ (some derivative of $f$ of an order $\leqslant N-1$ ).

The equilibrium theory (sections 14-16) requires no change.

In section 17: $1^{\circ}$ the inequality $N_{0} \leqslant c_{3} / \delta^{2}$ is to be replaced by $N_{0} \leqslant c_{3} \delta^{1-N}, 2^{0}$ when estimating $\left\|\alpha_{i}-\beta_{i}\right\|^{2}$, the condenser energy is $m_{i}^{2}\left(4^{N-2}-1\right) 4^{2-N} \delta^{2-N}$ instead of $3 m_{i}^{2} / 4 \delta$. So Theorem VI is obtained without any substantial change $\left(c_{4}\right.$ depends slightly on $\left.N\right)$ and so is Theorem VII, since still $|\delta C|_{N-1} \leqslant c_{N}^{\prime} \delta$ provided $|C|_{N-2} \leqslant \mid$ sphere of radius $\left.R_{0}\right|_{N-2}$, where the indices denote dimensions of both the indicated set and the volume meant by $\mid 1$.
In the proof of Lemma 8 , all goes as for $N=3$ till $T^{N-1}$ is obtained; $T^{k}$ means a set in the $u^{1}, u^{2}, \ldots, u^{k}$-space $T^{k}=\langle 0,4 \delta\rangle \times(a \quad k-1$-dimensional cube of side $l$ ). We have cap $\delta C \leqslant \operatorname{cap} T^{N-1}$. Denote by $\tau_{k}$ the $k$-dimensional volume measure in $T^{k}: \tau_{k} \subset T^{k}, d \tau_{k}=d u^{1} \ldots d u^{k}$. Remember that potentials accord in dimension with the whole space, the dimension of which is higher by 1 than that of integrals below. Write $u^{N-1}=\varrho \operatorname{tg} \theta, \varrho^{2}=\left(u^{2}\right)^{2}+\ldots+\left(u^{N-2}\right)^{2}, r^{2}=\varrho^{2}+\left(u^{N-1}\right)^{2}$. Then $\left[u^{1}, u^{2}, \ldots\right.$ $\left.\ldots, u^{N-2}, \varrho \operatorname{tg} \theta\right] \epsilon T^{N-1}$ at least if $\left[u^{1}, \ldots, u^{N-2}\right] \epsilon T^{N-2}$ and $|\theta| \leqslant \gamma_{N}$ $=\operatorname{arctg} l / \sqrt{(N-3) l^{2}}$. We have $d u^{N-1}=\left(\varrho / \cos ^{2} \theta\right) d \theta, \quad r=\varrho / \cos \theta$, so for $u \in T^{N-1}$

$$
\begin{aligned}
U^{\tau} N-1(u) & \geqslant U^{\tau} N-1(0, \ldots, 0) \\
& \geqslant \int_{T^{N-2}} d \tau_{N-2} \int_{-\gamma_{N}}^{\gamma_{N}} \frac{(\cos \theta)^{-N}}{\varrho^{N-3}} d \theta \\
& \geqslant 2 \gamma_{N}\left(\cos \gamma_{N}\right)^{-N} \int \ldots=A_{N} \int_{T^{N-2}} \frac{1}{\varrho^{N-3}} d \tau_{N-2} \\
& =A_{N} U^{\tau}{ }^{N-2}(0, \ldots, 0) .
\end{aligned}
$$

By induction

$$
U^{\tau} N-1(0, \ldots, 0) \geqslant A_{N}^{*} U^{\tau_{2}}(0,0) \geqslant A_{N}^{*} a,
$$

where $a$ has the same meaning as in (19.2) and $A_{N}^{*}=A_{N} A_{N-1} \ldots A_{4}$. Now the proof runs as in section 19. So, for $N \geqslant 4$ the theorem VIII is also valid.

\section{REFERENOES}

[1] N. Bourbaki, Intégration, Paris 1952.

[2] - Topologie générale, Paris 1940.

[3] M. Brelot, La théorie moderne du potential, Annales de I'Institut Fourier 4 (1954), p. $113-140$.

[4] H. Cartan, Théorie du potentiel Newtonien, Bulletin de la Société Mathématique de France 73 (1945), p. 74-106.

[5] - Théorie générale du balayage en potentiel Newtonien, Annales de l'Université de Grenoble 22 (1946), p. 221-280.

[6] 0. Frostman, Potentiel d'équilibre et capacité des ensembles, Comm. Sém. Math. Univ. Lund 3 (1935), p. 118.

[7] C. F. Gauss, Allgemeine Lehrsätze in Beziehung auf die im verkehrten Verhältnisse des Quadrats der Entfernung wirkenden Anziehungs- und Abstoßungs-Kräfte, Werke V. Berlin 1877, p. 195-242.

[8] O. Kellog, Fundations of potential theory, Berlin 1929, p. 384.

[9] W. Kleiner, Degree of approximation in Leja's extreme points method and its modification, Colloquium Mathematicum 11 (1964), p. 291-292. 
[10] M. Krzyżański, Sur les fonctions à variation bornée au sens de Hardy, Travaux de la Société des Sciences et des Lettres de Wilno, Classe des Sciences Mathématiques et Naturelles 12 (1939), p. 1-3.

[11] M. Ohtsuka, On potentials in locally compact spaces, Journal of Sciences of the Hiroshima University, Series A.I, 25 (1961), p. 135-152.

[12] J. Plemelj, Potentialtheoretische Untersuchungen, Leipzig 1911, p. 100

[13] M. O. Reade, On averages of Newtonian potentials, Bulletin of the American Mathematical Society 53 (1947), p. 321-331.

14] M. Riesz, Intégrales de Riemann-Liouville et potentiels, Acta Litterarum ac Scientiarum, Sectio Scientiarum Mathematicarum, Szeged, 9 (1938), p. 1-42.

JAGELLONIAN UNIVERSITY

Regu par la Rédaction le 15.3. 1963

J. SICTAK (CRACOVIE)

P 449. Formulé dans la communication A generalization of Schwarz's Lemma and of Hadamard's three circles theorem.

Ce fascicule, p. 206.

\section{W. KLEINER (CRACOVIE)}

$\mathbf{P} 450$ et 451. Formulés dans la communication On the equitibrium of signed measures. 